\title{
Web-based Collaboration in Flexible Engineering Education
}

\author{
Anh-Vu Nguyen \\ School of Engineering \\ Swiss Federal Institute of Technology (EPFL) \\ $\mathrm{CH}-1015$ Lausanne \\ Switzerland \\ +41216937338 \\ Anhvu.nguyenngoc@epfl.ch
}

\author{
Denis Gillet \\ School of Engineering \\ Swiss Federal Institute of Technology (EPFL) \\ $\mathrm{CH}-1015$ Lausanne \\ Switzerland \\ +41216935168 \\ Denis.gillet@epfl.ch
}

\begin{abstract}
This paper presents an innovative approach to sustain collaborative hands-on activities in flexible engineering education. The paper presents our research context, our objectives as well as some preliminary results. The main issues addressed include (i) object-oriented model for collaboration in Web-based learning environment for engineering education (ii) continuity of interaction in flexible engineering education (iii) awareness in Web-based learning environment, and (iv) evaluation methods and metrics. This doctoral research work is currently performed at the School of Engineering, EPFL.
\end{abstract}

\section{Categories and Subject Descriptors}

K.4 [Computer \& Society]: Organizational impacts Computer Supported Collaborative Work. H.5 [Information Interfaces \& Presentation]: Group and Organization interfaces - Computer Supported Cooperative Work, Evaluation/Methodology, Web-based interaction

\section{General Terms}

Measurement, Design, Experimentation, Human Factors.

\section{Keywords}

Collaboration, Web-based experimentation environment for engineering education, artifact, object-oriented model, continuity of interaction, awareness, evaluation.

\section{INTRODUCTION}

The rapid development of the Internet in the last decade has provided new possibilities and also new challenges for designing and deploying distance learning systems. Nowadays, one of the useful and interesting trends to support learning in engineering curriculum is to expand the available educational resources by providing virtual and real experimentation facilities [12]. Web-based experimentation turns to be a key feature in the deployment of e-Learning solutions for engineering education. It offers a tremendous opportunity to add flexibility in traditional curriculum by providing students with versatile access to the learning material from both a time and a location perspective $[12,22,28]$. The access to the lab resources is not restricted in fixed time slots as usually found in face-toface modalities. In fact, the flexible learning paradigm provides a solution for challenges posed to traditional academic institutions in many aspects, including pedagogical, technological, and organizational ones [12]. From a pedagogical perspective, flexible education means providing students with extended accessibility to learning resources, increased freedom to organize their learning activities and enhanced participation, autonomy and collaboration. From a technological perspective, flexible education corresponds to an adequate exploitation of the information and communication devices and infrastructures, especially the Internet. From an organizational point of view, flexible education relies on renewed study programs, regulations, as well as partnerships and collaborations with other institutions.

An important ingredient in engineering education is practical activities carried out through hands-on sessions. This introduces two requirements for building Web-based learning environments for engineering education.

- $\quad$ First, the Web-based experimentation environment must provide the students with interactive content for performing the experimental part of the work, allowing multiple trial-and-error cycles. This requirement refers to the hands-on activities. The Web-based hands-on approach relies on the benefits to be gained from alternative means of experimentation that allow the students to reinforce their understanding of physical phenomena in a framework where errors are neither penalized nor hazardous. The Web-based experimentation paradigm also helps to expand the diversity of education resources as well as to sustain the variety of learning resources that are provided to engineering students [12, 22, 35, 41]. As a consequence, the Web-based learning environment for engineering education should support the integration of heterogeneous Web components into the same environment. In other words, the Web-based learning environment should support the interaction between users and Web components as well as between different Web components.

- $\quad$ Second, the Web-based learning environment for engineering education must support collaborative activities since hands-on sessions are usually conducted in small 
groups. This requirement refers to the collaborative activities. Collaboration plays a very important role in knowledge building, sharing and distribution, especially in a flexible context where students can follow different learning modalities to perform multi-session experiments. The collaboration between students working actively in small groups can help them to work more productively in the laboratory and also learn more easily. The literature seems to be supporting that the social interaction amongst learners plays an important part in the learning process. In fact, it may have a significant impact on learning outcomes $[3,6,9]$.

Our work falls on the e-Learning multidisciplinary domain, where there is a cross feeding between computer and information sciences, and human sciences. It is mostly related to Computer Supported Cooperative Work (CSCW), HumanComputer Interaction (HCI), and Social Sciences (Social Network Analysis and Constructivism).

This paper presents briefly my dissertation work, started officially under Doctoral program in Computer, Communication, and Information Sciences at the Swiss Federal Institute of Technology in Lausanne (EPFL) in October 2003, under supervision of Professor Denis Gillet, head of the Sustainable Interaction Systems Group at the School of Engineering, EPFL. Section 2 presents the problems and our research context. Section 3 is about the research objectives. In section 4, we present our preliminary results. Section 5 concludes the paper.

\section{PROBLEMS AND CONTEXT FOR OUR RESEARCH AND DEVELOPMENT}

With help of CSCW, a wide variety of systems have been designed and developed to support collaborative and distance learning activities [46]. However, currently, most applications applied to e-Learning have involved expensive solutions to the problem of information delivery and have neglected the important aspects of interaction and collaboration among participants (in both user and system levels) in Web-based learning communities. A lot of problems still remain unsolved.

- Generally, most of CSCW applications do not support collaboration in Web-based flexible hands-on activities. To support synchronous collaboration, one can integrate different kinds of video-audio conferencing systems, such as Microsoft NetMeeting, or Flash Communications. However, in flexible learning, where asynchronous activities play a more important role, the applied CSCW applications are mostly restricted to the collection of documents into shared workspaces, such as BSCW [1], or restricted to the collaborative editing of text-oriented pages that may refer to external documents, for example Sparrow system [5] .

- There is no conceptual model that helps users, course designers as well as developers to understand the basic principles, such as main actors and their relationships, of collaboration process in Web-based experimentation learning environment from both social and technical points of view. The model should take into account both of these points of view, as we do believe that there is a strong relationship between the interaction and collaboration process in the user level and the interaction process in the system level in such kinds of learning environment.

- $\quad$ Flexible pedagogical scenarios introduce many sources of discontinuities of interaction (in both user and system levels). The concept of continuity of interaction is quite important in the context of flexible hands-on activities. The discontinuity of interaction prevents students from getting the sense of dealing with 'reality', which is normally well supported in face-to-face learning modalities. Such a discontinuity of interaction prevents clearly the collaboration between students. It also slows down and complicates the student experimental tasks.

- Evaluation issues: This is one of the important reasons why CSCW applications fail [14]. It is extremely difficult to evaluate a multi-user application. It is even harder to evaluate qualitatively and quantitatively the system acceptability $[33,36]$ in both social and practical acceptability, as well as to evaluate pedagogical performance in flexible education. Currently, it seems that there is no method that can be applied effectively and efficiently to evaluate the utility and usability of Webbased experimentation environment, as well as the flexibility, collaboration, and learning performance of students when performing the experiments using the environment.

In fact, in order to sustain the flexible learning deployment, a Web-based environment, namely eMersion, that supports handson experimentation through remote manipulation of physical laboratory devices and/or computer simulation tools has been developed at the School of Engineering, EPFL. The eMersion environment is currently used in Automatic Control, Fluid Mechanics and Biomechanics courses at the School of Engineering, EPFL. The environment provides the student with the possibility to carry out experimentation in a flexible way, i.e. students can follow different learning modalities [31] to perform multi-session experiments. The eMersion environment has a Cockpit-like user interface and contains all the components necessary to successfully complete laboratory assignments [13]. These components are heterogeneous in the sense that they were developed using different technologies and may be located on different servers. Figure 1 shows a normal lab session in which students work in the laboratory and interact with a teaching assistant (face-to-face modality). In face-to-face or flexible learning modalities, students use the same eMersion environment to perform their tasks. In average, 85 students from different engineering sections (Mechanical, Electrical and Micro engineering) enrolled in the Automatic Control course for each semester since the 2002 winter semester.

Our work has benefited from this rich context (with a real environment has been deployed and a significant number of students participated in the course using this environment), which helps us to clearly identify the problems in Web-based experimentation environment, as well as to develop, validate and refine our hypotheses and ideas.

We base our theoretical work on Activity Theory, which provides a broad conceptual framework for describing the structure, development, and context of computer-supported activities [11, 20, 44]. There is a thriving Activity Theory tradition in HCI studies in all over the world. We also explore 
the mediation role of artifact in Web-based engineering learning community, as we do believe that the artifact plays an important role in mediating the interaction process. Hands-on activities take place through and result in some kinds of artifacts. We are extremely interested in the metaphor of electronic laboratory journal, a special kind of artifact used by engineering students while performing their experiments. The laboratory journal, whose concepts and metaphor are fairly familiar with engineering students, takes a privileged place in engineering research and practices [27, 29]. We explore the role of shared laboratory journal in particular, and shared artifacts in general in a context of flexible learning in engineering education carried out through hands-on sessions. The Clover model [10] has been investigated as our conceptual and development framework for the groupware services and functionalities analysis. Social science theories such as Social Network Analysis [40, 45] and Constructivism theory [44] are explored as well. These theories would allow us to study, to understand the collaborative knowledge building process, to construct the social patterns, social relationships, and social structure in Web-based learning communities in engineering education.

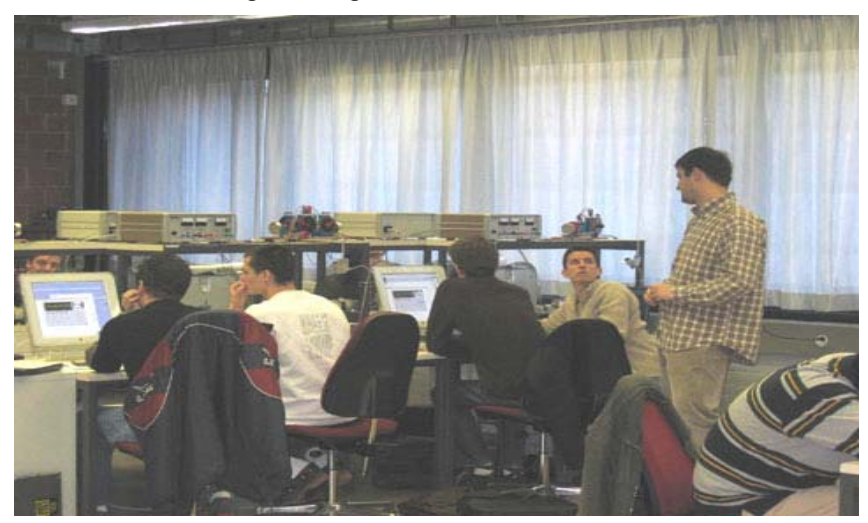

Figure 1. A face-to-face learning modality in Automatic Control course.

\section{OBJECTIVES}

Our main research challenge is to sustain the interaction and collaboration in Web-based experimentation environment for engineering education. The question is how a Web-based experimentation environment can provide engineering students with interaction and collaboration services, tools, or methods that are equally helpful in both face-to-face and flexible modalities? This question can be further investigated and opened to many smaller questions such as 'how to better fit a Web-based learning environment for hands-on activities', 'how to encourage flexible work', 'how to develop teamwork', and 'how to integrate a collaborative workspace into the flexible context of engineering education', etc.

This thesis is supposed to address the issues as follows

\section{Model}

The goal is to construct models for the interaction and collaboration process in flexible hands-on activities for engineering education, which are performed via a Web-based learning environment. The models may vary from the conceptual to the implementation, and to the deployment levels. The goal is to understand and thus to be able to identify the main actors as well as the relationships among these actors (in both user and system levels) participating in the interaction and collaboration processes. The aim of our models is to capture the domain characteristics, and to simplify the complexity of real work settings.

\section{Collaboration artifact}

The goal is to explore and develop methods that facilitate the flexible learning process in engineering education. This issue has resulted in our design and implementation of the concept of collaboration artifacts, which plays a crucial role in hands-on activities, as well as in our studies on various aspects of Webbased engineering learning communities such as participation, flexibility, and collaboration. The concept of collaboration artifact is investigated as a socio-technically mediated artifact (i.e. both from human look and from technical look or, in other words, from user and system levels). The theoretical focus is narrowed down to

\section{a. Continuity of interaction}

The notion of continuity has emerged as an objective that may potentially help users (refer to both professors and students) to obtain a higher quality of interaction, especially in a multimodality context. The continuity emphasizes the uninterrupted sequence of dialogue activities. In fact, the flexible learning modalities provide many sources for the discontinuities of interaction. Our goal is to analyze and define a multi-dimension framework related to the discontinuity, which is a base to provide theoretical as well as practical solutions for sustaining the continuity of interaction in a Web-based engineering learning community.

b. Services and metrics supplied by the artifact

- Sustaining, facilitating the collaboration: Our focus is almost on the generation and sustaining awareness information in Web-based experimentation environment when using the collaboration artifact. We find that the notion of awareness [8, 38] is particularly important and interesting with respect to our work on collaboration support.

- Evaluating the system acceptability and pedagogical performance. The metrics provided by the collaboration artifact when combined with traditional evaluation methods could be a solution for measuring the learning process, and for evaluating Web-based learning systems for engineering education. We propose a model for the evaluation issues. We study the (statistical) correlation between different independent and dependent variables defined by the collaboration artifact. This can help to understand, for instance, the impact of shared artifact on the learning outcomes.

\section{Prototype}

An extended electronic laboratory journal, namely eJournal, is developed. The eJournal serves as a collaboration artifact that implements our ideas and hypotheses previously presented. This means that the eJournal provides a shared workspace for users to interact and collaborate together, as well as for heterogeneous components within the same Web-based experimentation environment to 'share' and exchange data. More concretely speaking, the eJournal and its fragments are developed to 
sustain the continuity of interaction, to provide and sustain awareness, and to supply different evaluation metrics. Since the eJournal is used in real work settings, notably in various courses offered by the School of Engineering at EPFL, it will be an excellent framework to implement and validate our theoretical work.

As a summary, having dealt with the complexity of the Webbased experimentation environments, four areas for sustaining the interaction and collaboration are explored. The first one is related to an object-oriented model for collaboration in Webbased experimentation environment. The second explores the concept and framework of the continuity of interaction. The third explores the awareness issues. The fourth is concerned with the evaluation metrics and methods. We follow an iterative and participative approach for our research and development work, which are complement to each other. (i) The iterative approach encourages the prototyping and evaluating process. Parts of our theoretical work are prototyped in the eJournal functionalities. The environment is used by students from different engineering sections at EPFL. The evaluation feedbacks help us to validate and develop our ideas as well as to improve the prototype interface and functionalities. (ii) The participative approach focuses on the integration of users (students, assistants, professors, pedagogues, etc.) even from the conception process to allow them to express their ideas, their needs, as well as their experience.

In the next section we will present some of our preliminary results.

\section{PRELIMINARY RESULTS 4.1 Object-oriented model for collaboration in Web-based experimentation learning environment}

Interaction and collaboration in a Web-based experimentation environment are very complex processes. They include both technological and social elements. This means that they are composed of different sets of many interacting entities; interrelated through different levels, from social to system or technical ones. The user collaboration at the user level to realize an activity is in fact the source for the component interaction at the system level, which is supported by a relational database, a file system, and, a lot of services and tools. The result of the component interaction process is transformed into the information for users for other activities.

To create a model is an effective approach for analyzing and understanding a complex phenomenon [4]. Models and theories guide the designers as well as the developers from the conceptual phase to the development and the deployment phase by helping them to focus on the most relevant issues of the problems [15]. Cooperative models and theories have been successfully used in the development of CSCW systems. These models and theories represent the application domain and the most important aspects concerning the computer support of a cooperative activity [15]. They emphasize on coordination and organization aspects of work practices. One of most basic theories is Activity Theory, which guide our research as well as our development work. Other models and theories that should be considered include Coordination Theory [23], Object-oriented Activity Support model [43], Guareis de Frias's model [15].

Our aim is to create models that capture the Web-based interaction and collaboration process characteristics. The models not only present the most important actors and the relationships between these actors but also express our vision about the tightly couple between the social and the system levels. The models also present our proposed solution that is constructed around the concept of collaboration artifact.

In hands-on sessions, groups of students, teaching assistants and professors form different learning communities. In other words, a learning community is formed of various collaborative actors, which are in fact different groups or teams. A community has its own rules, norms, and conventions. Each user is a member of a group or team and has some particular roles and tasks depending on the division of tasks. Users perform their tasks by interacting with different objects in the Web-based environment, which represent components located on servers. Different objects at the user level represent different heterogeneous components. The components at the system are heterogeneous in the sense that they could be developed using different technologies and be located on different servers. The interactions between human actors and objects may change the status as well as behaviors of objects, which serve for the next activity of users. Human actors participate in an activity following a pre-defined or ad-hoc protocol. In fact, in order to obtain the (learning) outcome, students need to perform a chain of activities, in which the result of an activity may affect the next activities.

By taking a close look at both user and system levels, we identify the most important objects and the corresponding ones. This means that we identify the object with more or less similar roles but located in different levels. Human participants in the user level and Component sessions in the system level both play the role of Actors in the interaction process. Our vision is that both can interact with the same component, which serves as a 'bridge' between the user and system levels. This special component is what we call collaboration artifact. A collaboration artifact may consist of other collaboration artifacts. For instance, a laboratory journal is a collaboration artifact for engineering students. It contains different items, which also play the role of collaboration artifact since they also provide students with the collaboration possibilities.

The object-oriented model in Figure 2 shows the interaction and collaboration process in a Web-based experimentation environment. At the system level, the collaboration artifact is a kind of Web component, which is expressed by different Web component sessions. A Web component session can interact with other Web components. The interaction is supported by the session data generated from the collaboration artifact. A Web component is part of one or more objects visualized at the user level. At the user level, participants of the community also interact with the collaboration artifact. The artifact generates in this level the social data, which supports chains of activities. An activity is realized by chains of actions, which in turn carried out by different operations. Different participants participate in an activity. Participants are members of a community with the particular rules. Participants realize activities following the particular protocols. Participants in a community interact with objects following the division of labor, which may be divided 
into different settings. Participants can interact and collaborate with one another using different communication channels.

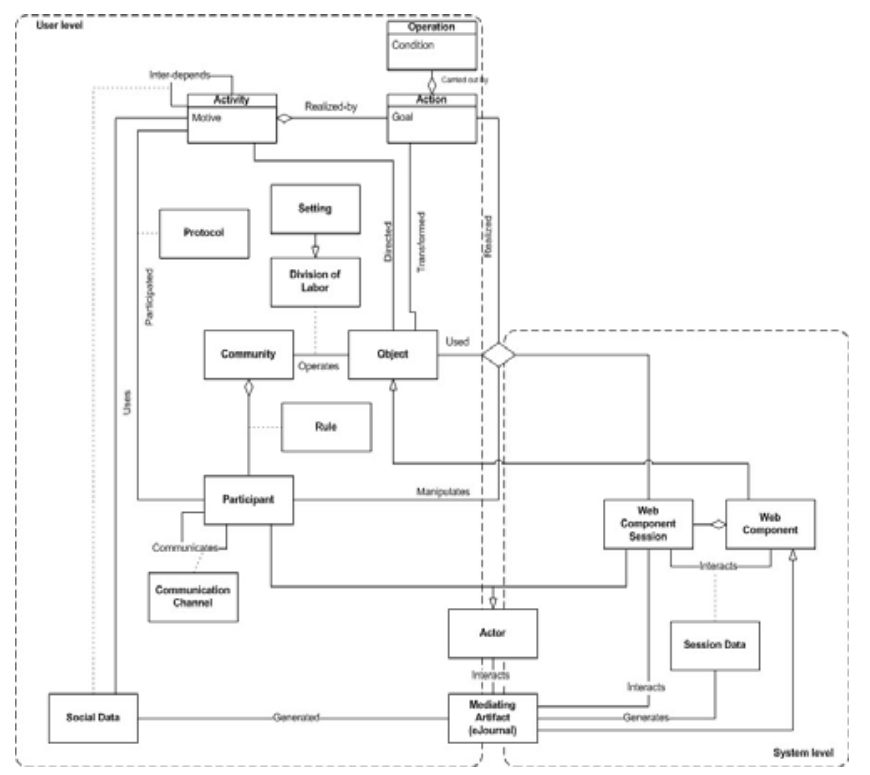

Figure 2. Conceptual model for interaction and collaboration process in Web-based experimentation env.

The proposed object-oriented model well presents the concepts from Activity Theory as well as clearly shows the mediation role of collaboration artifacts. It expresses the main actors and their relationships at different levels. It provides variety of concepts that helps users, course designers as well as developers to understand the basic principles of collaboration process in Web-based experimentation learning environment from both social and technical points of view. The model is however still at the conceptual level. We are currently extending the model by investigating other aspects such as object interaction, dynamic behaviors, collaboration constraints, etc.

\subsection{Continuity of interaction}

To improve and reinforce the collaboration among students performing the experiments, Web-based learning environments usually provide students with communication components such as forum, email or shared workspace. However, those components are normally poorly integrated together.

The notion of continuity has emerged as an objective that may potentially help users to obtain a higher quality of interaction, especially in the multi-session context as in the eMersion environment. The continuity emphasizes the uninterrupted sequence of dialogue activities. In other words, it highlights the importance of uninterrupted flow of information between the user and the interactive spaces [37]. From the point of view of humans [26] in engineering education, i.e. when performing the experiment using an object, a continuous interaction is one in which users can observe the behavior of that object, can make inferences about its state, and the state of any tasks that they are executing, and crucially, can issue commands to the object at any point, without needing to re-enter into any preparatory or enabling tasks to prepare the object. Continuity also means that the effects of changes are predicted or foreseen [25].
Flexible pedagogical scenarios introduce many sources of discontinuities of interaction. The discontinuity of interaction prevented clearly the collaboration between students. It also slowed down and complicated the student experimental tasks.

We have synthesized and defined different dimensions of continuity and the causes of discontinuity as follows

- $\quad$ Space: students perform tasks in many different consoles within the same experimentation environment. One should recall that one component (i.e. a tool integrated into the environment) is launched in at least one separate console. And an experimentation environment should integrate different components to allow students to complete successfully their experimental assignments. Students may also use external tools (or applications) to support their tasks. Obviously, performing the same task while opening different consoles may create the discontinuity of interaction.

- Place: the sense of place is a context understanding of the appropriateness of styles of behavior and interaction [18]. It refers rather to the physical places with embedded context understanding. Obviously, the behavior of users when performing the experiment in the laboratory and at home is not the same.

- Time: the segmentation of a hands-on session into multiple short sessions creates multiple time intervals clearly increases the potential mismatch between human and system capabilities.

- Cognition: this dimension covers and explains the three other ones. This dimension takes focus on the cognitive processes of the human as well as the states and processes of the performed task. As presented in [26], the context within which an information stream is being used determines the way that human will perceive it. The authors implied that to sustain the continuity of interaction, it is necessary for the system to encode or represent interactions in the same hierarchical manner as the user.

In a distributed integrated environment like the eMersion, students have to switch between different spaces, different places, at different times to perform even a single task. As explained by the Suchman's Situated Action Theory [42], the task performance is always situated action, determined by local and unanticipated events. Massink stressed this perspective by showing that the reaction depends on the particular situation, the experience and the knowledge of the user [25]. As a consequence, the user's perceptual models may change regularly in a multiple context situation, and may not match the user's conceptual models, which are built towards the overall goals.

We do believe that there is a strong relationship between different actors from different levels (user and system levels). This opinion is also supported by Massink [25], who stated that the continuous interaction implies a tighter coupling between user and system. In our approach, we propose the collaboration artifact, which serves as a 'bridge' that connects these two levels. The collaboration artifact provides a 'shared workspace' for both users and Web components. Data for group is stored in the space. As a shared workspace, the collaboration artifact allows students to collaborate with peers, not only with mates in 
the same group, but also with ones in other groups. The collaboration between professors, assistants and students are supported as well. At the system level, the collaboration serves also as a shared space for different component to exchange data. Technically, the collaboration artifact provides a data homogenization and transformation process that allows heterogeneous components to 'speak' or to interact with each other.

This new mechanism augments a lot the interaction process. It helps to sustain the continuity of interaction in Space, Place and Time dimensions. We try to overcome the discontinuity in Cognition by designing a homogeneous interface and by reducing the task complexity (by introducing more 'scripted' procedures to help students perform a task). Data are passed smoothly and naturally from one component to another. The requirement to use external applications for data sharing and exchanging is minimized. Users work with minimum discontinuity in all dimensions of interaction. As a consequence, the quality of the hands-on and collaborative works is much more improved. More discussions about the continuity of interaction could be found in [30].

\subsection{Awareness in Web-based learning environment}

Knowing the activities of other co-workers is a basic requirement for group interaction, which is the visible aspect of collaborations [24]. In a face-to-face condition, users find it naturally easy to maintain a sense of awareness about the activities of others. However, in other conditions, supporting spontaneous interaction is evidently much more difficult. To support effective collaboration, systems should provide group awareness, which is defined as 'an understanding of the activities and progresses of others, which provides a context for your own activities' [8]. Awareness of other group members is a precondition for interaction, a critical building block in the construction of team cognition, and consequently that computational support for awareness in groupware system is crucial for supporting team cognition in distributed groups [16]. In learning, awareness plays a very important role in facilitating the learning process, especially in a flexible context such as ours. Professors need awareness to have a general view of the class activities, to monitor the class progress, to detect problems in order to intervene in time. Students need awareness to have a view about their progress compared to other groups. Awareness is also necessary for students to find potential collaborators for exchanging documents and ideas, and to ask for help.

The concept of awareness plays the same important role in the Web-based learning community. So far, various awareness mechanisms have been produced to support group awareness [17], such as tele-pointers, radar-views, or distortion-oriented lenses. In fact, several systems with their awareness mechanisms have been design for supporting communities working on shared workspaces built on top of the WWW infrastructure. Generally speaking, all systems, which support working in groups, should provide a mechanism to gather data about the student's interaction, and shows the visualization of this information to the user. It is then up to users to interpret the awareness and decide which actions (if any) to take [19]. However, conventional awareness mechanisms do not cover all aspects of group collaboration in a flexible learning context. In other words, those mechanisms are not enough for monitoring the learning process in a Web-based learning environment. Such mechanisms do not always provide sufficient awareness about social structures, relations, memberships, as well as user roles; for instance with the information obtained, it is hard to answer the questions such as which tasks have been completed and when, which groups are more active than others, what are the relationships between the groups, which learning modalities are preferable to groups, is there any educational outcome difference between the groups who prefer working remotely and ones who prefer the face-to-face learning modalities, etc. In fact, so far, all Web-based experimentation environments do not provide at all or do not provide enough awareness for collaboration in a flexible context.

One of the important phases of the monitoring process is to select one or more high-level variables, termed indicators, which can represent some states of collaboration among users in the learning community. We suggest a new approach to provide awareness based on the visualization of user activities, which are related to the exchange of artifacts. The artifact-based interaction is aggregated into indicators and then displayed to the users. Different Social Network Analysis (SNA) methods are applied to construct the social structure and to find the interaction patterns in the learning community. In reality, many works have demonstrated the usefulness of SNA for the study of interaction among different groups [24, 34]. Their works, however, only applied at the evaluation phase for the study of the participatory aspect of learning. SNA [40, 45] is an approach that focuses on the study of patterns of relationships between actors in communities. The SNA issues are located in the intersection of the sociometry, group dynamics, graph theory, and anthropology domains. Using SNA methods, one would seek to model the relationship that depicts the structure of the community. So one could then study the impact of this structure on the functioning of the groups within the same community.

We have studied and developed various services that provide awareness about group activities in the learning community. Besides the availability awareness such as the user presence (who is currently connected to the environment), the user location (in the lab, at school, or at home), we also provide group awareness based on the artifact-related activities, which is richer than conventional artifact feedback or feed-through [7] at group and especially at community levels. The shared artifacts serve at the same time as a product and as a medium of the collaboration and the learning processes. The artifact, which is the experimental results or data used for hands-on activities, are collected to the shared workspace and shared among users. The artifact could reflect the student hands-on activities and student interactions in the environment. It also provides users with several ways to collaborate with each other. In fact, based on the concept of artifacts, which plays a critical role in hands-on activities, one could use different methods, different approaches to develop different services that provide awareness to facilitate the teamwork. We are extremely interested in different measures provided by the Social Network Analysis, which allow us to get awareness information about the collaboration and interaction activities in the environment. These measures in fact can be automatically calculated from the artifacts stored in the workspace. 


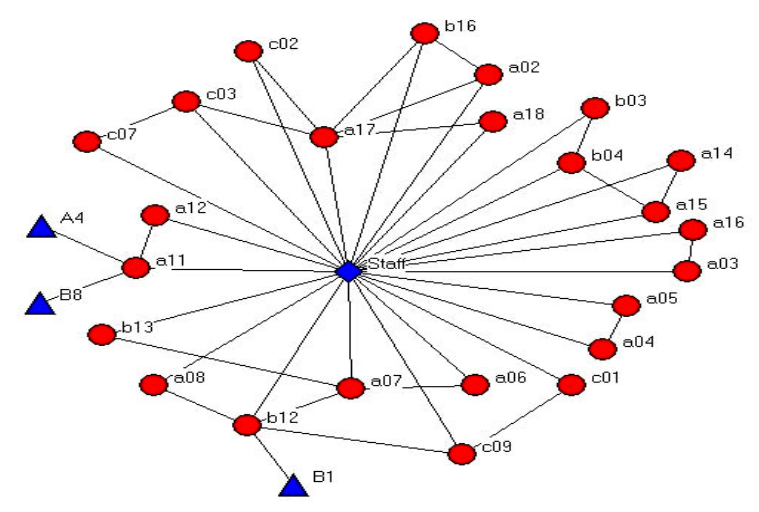

Figure 3. Sociogram for group interactions

As an example, Figure 3 is a sociogram representing the social structure of group collaborations. This sociogram is for the 2003 summer semester at the Automatic Control laboratory course at the EPFL. The sociogram is generated from the group-by-group matrix $\mathrm{NxN}$ ( $\mathrm{N}=$ total number of groups), where xij represents the fact that there is an interaction between the group at the ith row and the group at the jth column. In the sociogram, nodes (red circles) represent groups and lines represent the interaction between groups. We use different shapes and colors to refer to some special groups, the Staff group, i.e. the group of assistants who evaluate the students" works, represented by the blue diamond, and some groups from the previous semesters (A4, $\mathrm{B} 1, \mathrm{~B} 8)$ represented by the blue triangle. This event is fairly interesting. It indicates that there are relations between different groups enrolled in different academic years. This means that, for example, a group of the current semester can contact and receive copies of experimental data from another group from the previous semester. Other interesting measures include cliques, Freeman's centrality degree, etc.

The social network measures give professors and students a general overview of active and passive groups in the learning community, as well as the structure of the community. This is what we call 'social structure awareness'. For example, one can see clearly in Figure 3 that Mt-a17 is one of the most active groups, which plays an important role in the knowledge distribution in the class. Then, professors and assistants can use the obtained information to decide what to do next; for instance, the professor can re-organize the class structure to facilitate the student learning process. Students can find their positions in comparison to the whole class, so they can be more motivated. They can also find the potential groups with which they can collaborate. This kind of awareness information could also be useful to professors for understanding some student behaviors. For example, by seeing the chart representing the number of artifacts created by each student group, we have noted the fact that students work harder before the assignment due date, especially before the laboratory test (the course exam). More discussions about the awareness issues could be found in [32].

\subsection{Evaluation methods and metrics}

To date, very few studies have been performed to determine the effectiveness of the Web-based environments in engineering education [35]. Lessons learned from various Web-based learning environments as well as our experience gained from 4 academic semesters of deploying the eMersion environment reveal the difficulties associated with the introduction of the Web-based learning environment for engineering education. Studying and assessing the Web-based learning environment is one of the crucial fields in different research domain including Computer supported collaborative learning, Human-computer Interaction, etc. In single-user applications, it is already difficult to test the perceptual, cognitive, motor variables that have been the focus [23]. It is however extremely difficult to evaluate the multi-user applications [14], especially to evaluate the Webbased environment that supports hands-on activities where many interactions take place in both technical and social levels.

We have proposed a model, called Instrumentation Feedback Model for Assessment, which is illustrated in Figure 4. The term Instrumentation Feedback Model was coined and explained in the work of Leifer [21]. This term is used in the sense of observing both independent and dependent variables in an automatic feedback control environment. Our model includes 4 instrumentation Nodes. Each one represents a phase in the teaching and learning process using a learning environment. The outcomes are differentiated into different levels and each is assessed and validated through a feedback path. The output of the assessment process at one Node could provide feedbacks or could influence on the input of another Node.

The input of the whole process is a pedagogical scenario. It is important to integrate the design and development process around scenarios. Scenarios have people in them, they are specific, they are grounded in the real world, they describe an existing or envisioned system from the perspective of participated and non-participated users, including a narration of their goals, plans, and reactions [36]. The scenario is defined depending on the requirements on each course, the logistic matters of each department, etc. At Node 1, the pedagogic objectives, the course requirements are defined. Based on these definitions, the course environment is designed or re-designed. By re-design, we mean that some fundamental concepts of the environment has been modified or replaced. At Node 2, the professors and students requirements are defined in greater detail. The system functionalities that facilitate the teaching and learning process are also specified.

The assessment process is applied at Node 3 and Node 4. For the inner most, formative assessment loop at Node 3 takes place during the course. The goal of formative assessment is to identify aspects of the system that can be improved, and to provide guidance in how to make changes to a design. One big constraint in applying formative assessment is that it must not disturb the students who are currently using the system. Thus in general only minor modifications on the system functionalities are allowed. The summative assessment loop at Node 4 is aimed at measuring the acceptability of the system. According to Nielsen [33], system acceptability is achieved by meeting the social and practical acceptability. An important factor in practical acceptability is usefulness, which constitutes usability and utility, where utility is the question of whether the functionality of the system in principle can do what is needed, and usability is the question of how well users can use that functionality. 


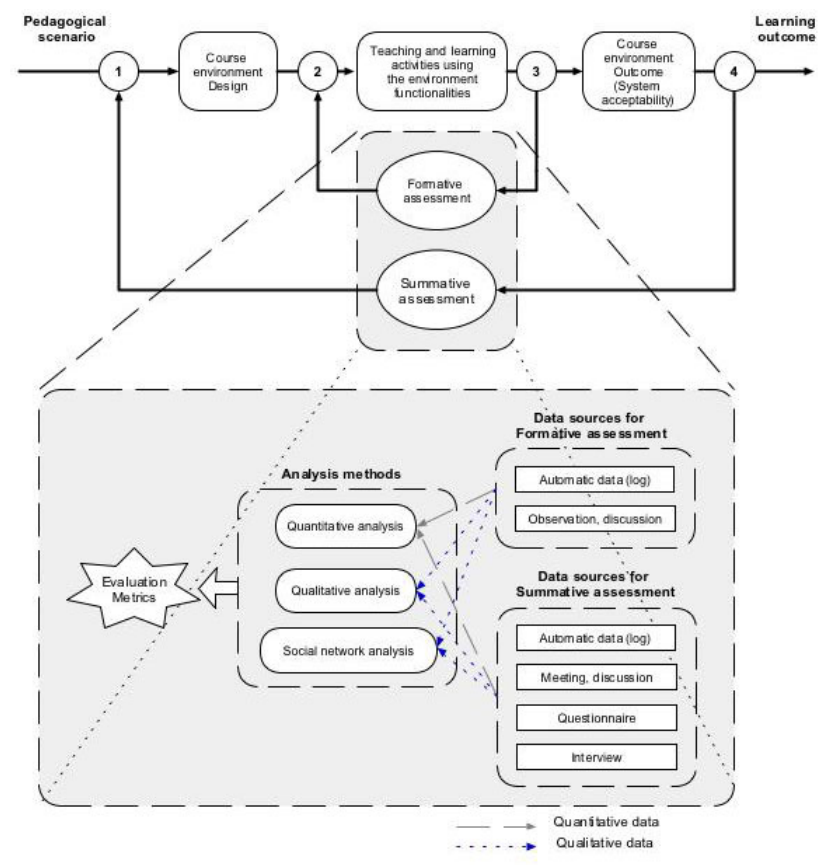

Figure 4. Instrumentation Feedback Model for Assessment

Let's take a closer look at the assessment methods. In the proposed model, all the analysis methods are fed with data coming from different sources, meeting the need for capturing different forms of interaction in a Web-based learning environment. For the formative assessment, the basic instruments providing data are automatic data coming from log, observation, and discussions directly with students and teaching assistants during the hands-on sessions. For the summative assessment, data sources come from automatic data (log), meeting, discussion, questionnaire, and interview. In fact, the log data is a convenient source, which can be used for data collection, for actions evaluation and feedback can be made available immediately to the learning community [34]. Especially in a hands-on environment, where different kinds of artifacts are at the same time instruments and results of the interaction and collaboration, the logged artifact-based actions can supply a great source to reflect the student hands-on activities and student interactions in the environment.

The analysis methods include quantitative, qualitative and social network analysis. Qualitative analysis provides a context to understand core usability issues. Qualitative data is used to interpret and explain what happened. Quantitative analysis is used to account for the occurrence of actions, thus helps to predict and measure some particular phenomena. Quantitative analysis facilitates the interpretation process used in qualitative analysis, and vice versa. Social network analysis methods are applied to construct the social structure and to find the interaction patterns in the learning community. Of course, the choices of assessment methods may not be the same. It depends on the pedagogical scenario as well as the assessment objectives.

The results of the analysis processes are selected, and aggregated to different evaluation metrics, which allow assessing the pre-defined evaluation objectives.
The shared artifacts logged supply different metrics allowing the observation of different dimensions of the Web-based learning process in engineering education. The first dimension is the amount of the students' work that takes place within the environment compared to work that occurs outside. This metric can be measured by calculating the correlation between the artifacts, which were created using tools integrated into the environment, and those that were created by an external tool and then uploaded to the environment. It refers to the utility of the environment for performing hands-on tasks. The second dimension is linked to the importance of flexible learning modalities compared to traditional face-to-face learning modalities. The metric can be measured by calculating the ratio of artifacts, which were created during flexible sessions, for example. Another dimension refers to the collaboration aspect. Other metrics are also defined to measure the influence of shared artifacts on the student learning performance; for example, to measure the correlation between the number of created artifacts and the students' grades.

We have carried out an evaluation process from the 2002 winter semester up to now. The assessment results open up a new set of ways for assessing the flexible and collaborative work in a learning environment for engineering education. The proposed Instrumentation Feedback Model for Assessment was generalized from and validated by the experience done during these 4 semesters. Although the model is used for assessing the Automatic Control laboratory courses at the EPFL, it is general enough to apply to any other pedagogical scenario, or any other learning system. A key point is that it follows an iterative process, through different assessment loops. These assessment loops allow the developer and evaluator intervene in time to adapt the system to the requirements from users or the requirements arise from a new input pedagogical scenario. Another point is that the model uses an approach in which different analysis methods are mixed. These analysis methods are fed with data coming from different sources, meeting the need for capturing different forms of interaction in a Web-based learning environment. More discussions about the evaluation issues could be found in [31] .

\section{CONCLUSION AND FUTURE WORK}

This paper presents our research context, problems and objectives. The main research question is how to support teamwork for flexible hands-on activities in engineering education. Our proposed approach is based on the concept of collaboration artifact that mediates the collaboration.

In this paper, we briefly discuss about the preliminary results of our research. The main contributions include an object-oriented model for collaboration in Web-based experimentation learning environment, a conceptual framework related to the continuity of interaction. We also discuss the notion of awareness in this kind of learning environment as well as a new approach for providing awareness information. Based on the notion of collaboration artifacts, one can develop different kinds of tools and services, which provide awareness about the group activities, group progresses, and the social structure of the community.

The choice of some classical usability engineering methods has proved useful when combined with clear measures of student's 
activities based on the use of collaboration artifacts for collecting and sharing experimental data in hands-on activities. We propose a model for the iterative assessment, in which the feedback from the users plays an important role. The assessment model also facilitate different analysis methods such as quantitative, qualitative and social network analysis, which meet the need for capturing different forms of interaction in a Webbased learning environment. In doing so, we hope that this contribution has illustrated the possibility to overcome the challenges of the evaluation of Web-based learning systems.

We follow the participative and iterative approach, in which the user feedbacks and our observations help to validate and develop our hypotheses and models.

We would like to continue and extend our studies on the impact of collaboration artifacts in an engineering learning community, especially in a flexible context. The role of collaboration artifact in engineering education is an open research topic to be investigated. From the researcher's point of view, it could be considered as an instrument to measure the participation, flexibility and collaboration in a learning community. It helps to mediate and measure the group activities. It also takes the role of articulation artifact [39], or collaboration artifact as defined in [2]. From the professor and student's point of view, it could be used to construct group mirrors [19] or many kinds of group awareness. From the designer's point of view, it could be used to check the acceptability and utility. From the pedagogical point of view, it could help to introduce different learning scenarios. Using this kind of artifacts, there are also possibilities to define different independent and dependent variables for the study of influence of different conditions on the learning scenario.

\section{ACKNOWLEDGMENTS}

This work is partially funded by the Board of the Swiss Federal Institutes of Technology in the framework of its New Learning Technologies program, and partially funded by the ProLEARN Network of Excellence, a project of European Commission.

\section{REFERENCE}

[1] Appelt, W. and Mambrey, P., Experiences with the BSCW Shared Workspace System as the Backbone of a Virtual Learning Environment for Students. in World Conference on Educational Multimedia, Hypermedia and Telecommunications, (Seattle, USA, 1999), 1710-1715.

[2] Bardram, J. Collaboration, Coordination, and Computer Support - An Activity Theoretical Approach to the Design of Computer Supported Cooperative Work, PhD thesis University of Aarhus, 1998.

[3] Berge, Z.L. and Collins, M. Computer-Mediated Communications and the Online Classroom: An Introduction. in Berge, Z.L. and Collins, M. eds. Computer Mediated Communication and the Online Classroom, Hampton Press Inc., New Jersey, 1995.

[4] Boman, M., Bubenko, J.A., Johannesson, P. and Wangler, B. Conceptual Modeling. Prentice Hall, 1997.
[5] Chang, B.W., In-place editing of web pages: Sparrow community-shared documents. in WWW7 Conference, (Brisbane, Australia, 1998).

[6] Dillenbourg, P., Baker, M., Blaye, A., O Malley, C. The evolution of research on Collaborative learning. in Spada, E. and Reiman, P. eds. Learning in Humans and Machine: Towards an interdisciplinary learning science, Elsevier, Oxford, 1995.

[7] Dix, A., Finlay, J., Abowd, G. and Beale, R. Human Computer Interaction. Prentice Hall, 1993.

[8] Dourish, P. and Bellotti, V., Awareness and Coordination in shared workspaces. in Proceedings of CSCW, (Toronto, Canada, 1992), 107-114.

[9] Eastmond, D. and Ziegahn, J. Instructional Design for the Online Classroom. in Berge, Z.L. and Collins, M. eds. Computer Mediated Communication and the Online Classroom, Hampton Press Inc., New Jersey, USA, 1995.

[10] Ellis, C. and Wainer, J., A Conceptual Model of Groupware. in Proceedings of ACM Conference on CSCW, (Chapel Hill, NC, USA, 1994), ACM Press.

[11] Engeström, Y. Learning by expanding. An activitytheoretical approach to developmental reserarch. Helsinki: Orienta-konsultit, 1987.

[12] Gillet, D. Towards flexible learning in engineering education. in Innovations - 2003: World Innovations in Engineering Education and Research, iNEER in Cooperation with Begell House Publishers, 2003, 95-102.

[13] Gillet, D., Geoffroy, F., Zeramdini, K., Nguyen, A.V., Rekik, Y. and Piguet, Y. The Cockpit, An effective Metaphor for Web-based Experimentation in Engineering Education. International Journal of Engineering Education, 19, No. 3. 2003, 389-397.

[14] Grudin, J., Why CSCW applications fail. in Proceedings of ACM Conference CSCW, (Porland, Oregon, USA, 1988), 95-93.

[15] Guareis de Farias, C.R., Pires, L.F. and Van Sinderen, M., A conceptual model for the development of CSCW systems. in Proceedings of the 5th International Conference on the Design of Cooperative Systems, (France, 2000), 189-204.

[16] Gutwin, C., Greenberg, S. The importance of Awareness for Team Cognition in Distributed Collaboration. APA Press, 2004.

[17] Gutwin, C., Roseman, M. and Greenberg, S., A Usability study of Awareness widgets in a shared workspace groupware system. in Proceedings of CSCW, (Massachusetts, USA, 1996), 258-267.

[18] Harrison, S. and Dourish, P., Re-Place-ing Space: The Roles of Place and Space in Collaborative Systems. in Proceedings of CSCW, (New York, USA, 1996), 67-76.

[19] Jermann, P., Soller, S. and Muehlenbrock, M., From Mirroring to Guiding: A Review of State of the Art Technology for Supporting Collaborative Learning. in Proceedings of ECSCL, (Maastricht, The Nertherlands, 2001). 
[20] Kuutti, K. Activity Theory as a potential framework for Human-Computer Interaction Research. in Nardi, B.A. ed. Context and Consciousness: Activity and Human-Computer Interaction, The MIT Press, London, England, 1995.

[21] Leifer, L. Design Team performance: Metrics and The impact of Technology. in Evaluating Corporate Training: Models and Issues, Kluwer, 1997.

[22] Magin, D.e.a. Engineering Students' Understanding of the Role of Experimentation. European Journal of Engineering Education, Vol. 83 No. 3. 2000, 351-358.

[23] Malone, T.W. and Crowston, K., What is Coordination Theory and how can it help design cooperative work systems? in Proceedings of the ACM Conference on CSCW, (Los Angeles, California, USA, 1990).

[24] Martínez, A., Dimitriadis, Y., Rubia, B., Gómez, E., Garrachón, I. and Marcos, J.A., Studying social aspects of computer-supported collaboration with a mixed evaluation approach. in Proceedings of CSCL, (Boulder, Colorado, USA, 2002).

[25] Massink, M. and Faconti, G. A reference framework for continuous interaction. Journal of Universal Access in the Information Society. 2002, 237-251.

[26] May, J., Buehner, M.J. and Duke, D. Continuity in cognition. Journal of Universal Access in the Information Society. 2002, 252-262.

[27] McCormack, J.B., Morrow, R.K., Bar, H.F., Burns, R.J. and Rasmussen, J.L. The complementary Roles of the Laboratory Notebooks and Laboratory Reports. IEEE Transactions on Education, Vol. 34, No.1., 2001

[28] Mosterman, P., Dorlandt, A.M., Olin Campbell, J., Burow, C., Bouw, R., Brodersen, A.J. and Bourne, R. Virtual Engineering Laboratories: Design and Experiments. Journal of Engineering Education, Vol. 83 No. 3. 1994, 279-285.

[29] Myers, J.D., Fox-Dobbs, C., Laird, J., Le, D., Reich, D. and Curtz, T., Electronic Laboratory Notebooks for Collaborative Research. in Proceedings of the 5th Workshop on Enabling Technologies: Infrastructure for Collaborative Enterprises, (Stanford, USA, 1991), 47-51.

[30] Nguyen, A.-V., Gillet, D., Rekik, Y. and Sire, S., Sustaining the continuity of interaction in Web-based Experimentation for Engineering Education. in Proceedings of the International Conference on Computer Aided Learning in Engineering Education, (Grenoble, France, 2004).

[31] Nguyen, A.-V., Gillet, D. and Sire, S. Evaluation of a Webbased learning environment for Hands-on experimentation. in Innovations 2004: World Innovations in Engineering Education and Research, iNEER in Cooperation with Begell House Publishers, New York, USA, 2004, 303-315.
[32] Nguyen, A.-V., Gillet, D. and Sire, S., Sustaining Collaboration within a learning community in enginering education. in World Conference on Educational Multimedia, Hypermedia, and Telecommunications, (Lugano, Switzerland, 2004), AACE.

[33] Nielsen, J. Usability Engineering. Morgan Kaufmann Publishers, 1993.

[34] Nurmela, K., Lehtinen, E. and Palonen, T., Evaluating CSCL Log files by social network analysis. in Proceedings of CSCL, (Stanford, California, USA, 1999).

[35] Ogot, M., Elliott, G. and Glumac, N. An assessment of Inperson and Remotely Operated Laboratory. International Journal of Engineering Education. 2003, 57-64.

[36] Rosson, M.B., Carroll, J.M. Usability engineering scenario-based development of human-computer interaction. Morgan Kaufmann, 2002.

[37] Savidis, A., Maou, N., Pachoulakis, I. and Stephanidis, C. Continuity of interaction in nomadic interfaces through migration and dynamic utilization of I/O resources. Journal of Universal Access in the Information Society. 2002, 274287.

[38] Schmidt, K. The problem with 'awareness': Introductory remarks on 'awareness in CSCW'. Computer Supported Cooperative Work (CSCW). The Journal of Collaborative Computing, Vol. 11 No. 3. 2002, 285-298.

[39] Schmidt, K. and Bannon, L. Taking CSCW seriously: Supporting articulation work. CSCW: The Journal of Collaborative Computing, Vol. 1, no. 1.1992

[40] Scott, J. Social Network Analysis, a handbook. SAGE Publications Ltd., London, UK, 2001.

[41] Starr, D. Virtual Education: Current practices and future direction. The Internet and Higher Education, Vol 15. 1998, 420-425.

[42] Suchman, L.A. Plans and situated actions, the problem of human machine communication. Cambridge University Press, 1987.

[43] Teege, G. Object-oriented Activity Support: A model for Integrated CSCW systems. Computer Supported Cooperative Work (CSCW): The Journal of Collaborative Computer, Vol. 5 No. 1. 1996, 93-124.

[44] Vygotsky, L.S. Mind in Society. The development of Higher Psychological Processes. Harvard University Press, London, UK, 1978.

[45] Wasserman, S. and Faust, K. Social Network Analysis: Methods and Applications. Cambridge University Press, 1994.

[46] Xu, C. Interaction and Collaboration mechanisms for Distributed Communities and Groups in Educational Settings, PhD thesis. Institut fur Informatik der Technischen Universitat Munchen, Munich, 2000. 